Discrete Comput Geom 29:575-593 (2003)

DOI: $10.1007 / \mathrm{s} 00454-002-0788-\mathrm{x}$

Geometry

\title{
Mixed Polytopes
}

\author{
Rolf Schneider \\ Mathematisches Institut, Albert-Ludwigs-Universität, \\ D-79104 Freiburg i. Br., Germany \\ rolf.schneider@math.uni-freiburg.de
}

\begin{abstract}
Goodey and Weil have recently introduced the notions of translation mixtures of convex bodies and of mixed convex bodies. By a new approach, a simpler proof for the existence of the mixed polytopes is given, and explicit formulae for their vertices and edges are obtained. Moreover, the theory of mixed bodies is extended to more than two convex bodies. The paper concludes with the proof of an inclusion inequality for translation mixtures of convex bodies, where the extremal case characterizes simplices.
\end{abstract}

\section{Introduction}

In recent years, there has been increasing interest in extending parts of the classical integral geometry of the motion group in Euclidean spaces to the group of translations (see [2], [3], [5], [6], [10], and [12]-[17]). This was partially motivated by possible applications to the stochastic geometry of homogeneous random geometric structures. At the same time, the scope of functionals for which integral-geometric intersection formulae can be obtained has been widened considerably. An example is the recent work of Weil [16] and Goodey and Weil [3] on translative integral geometry of support functions, by which the present paper was inspired. For convex bodies $K, M$ in Euclidean space $\mathbb{R}^{n}$ and for the centred support function $h^{*}$ (the support function with respect to the Steiner point), Weil [16] has proved the formula

$$
\begin{aligned}
\int_{\mathbb{R}^{n}} h^{*}(K \cap & (M+x), \cdot) d x \\
& =V_{n}(M) h^{*}(K, \cdot)+\sum_{j=2}^{n-1} h_{j}^{*}(K, M, \cdot)+V_{n}(K) h^{*}(M, \cdot),
\end{aligned}
$$

where the integration is with respect to Lebesgue measure and $V_{n}$ denotes the volume of convex bodies. The function $h_{j}^{*}(K, M, \cdot)$ is homogeneous of degree $j$ in $K$ and of 
degree $n+1-j$ in $M$. Of the facts proved in [16] about these functions, we mention only their explicit representation in the case of polytopes that is given by the case $k=2$ of (9) below.

It is clear that the left-hand side of (1) defines the support function of a convex body. This body is denoted by $T(K, M)$ and called the translation mixture of $K$ and $M$. In [3] Goodey and Weil were able to prove that the functions $h_{j}^{*}(K, M, \cdot)$ are also support functions. Hence, for the translation mixture $T(K, M)$ there is a polynomial expansion

$$
T(\lambda K, \mu M)=\sum_{j=1}^{n} \lambda^{j} \mu^{n+1-j} T_{j}(K, M)
$$

for $\lambda, \mu \geq 0$, with convex bodies $T_{j}(K, M)$, called mixed bodies of $K$ and $M$. This reminds one of the fact that the integral analogous to (1) given by

$$
\int_{\mathbb{R}^{n}} \chi(K \cap(M+x)) d x=V_{n}(K-M),
$$

where $\chi$ denotes the Euler characteristic, also has a polynomial expansion, namely

$$
V_{n}(\lambda K-\mu M)=\sum_{j=0}^{n} \lambda^{j} \mu^{n-j}\left(\begin{array}{l}
n \\
j
\end{array}\right) V(K[j],-M[n-j])
$$

yielding the mixed volumes of two convex bodies and thus leading into a very rich theory. It is hoped that a further development of the theory of mixed bodies will reveal some similarly interesting features.

When talking in the following of mixed polytopes, it should be noted that objects with this name have already appeared in the literature, see p. 953 of [4]. As the latter are not polytopes, but elements of the polytope algebra, there is no danger of confusion.

In the present paper, we first point out the analogy of the translation mixtures of polytopes with the fibre polytopes introduced by Billera and Sturmfels [1]. Our main aim then is the treatment of mixed polytopes by a new approach, in part using ideas from [9]. This not only allows us to give a simpler proof for the fact that the functions $h_{j}^{*}(K, M, \cdot)$ are support functions, but also permits an easy extension to translation mixtures of more than two convex polytopes. For polytopes $P_{1}, \ldots, P_{k}(k \geq 2)$ and for $\lambda_{1}, \ldots, \lambda_{k} \geq 0$ there is an expansion

$$
\begin{gathered}
\int_{\mathbb{R}^{n}} \cdots \int_{\mathbb{R}^{n}} h^{*}\left(\left(\lambda_{1} P_{1}+x_{1}\right) \cap \cdots \cap\left(\lambda_{k-1} P_{k-1}+x_{k-1}\right) \cap \lambda_{k} P_{k}, \cdot\right) d x_{1} \cdots d x_{k-1} \\
=\sum_{\substack{m_{1}, \ldots, m_{k}=1 \\
m_{1}+\cdots+m_{k}=(k-1) n+1}}^{n} \lambda_{1}^{m_{1}} \cdots \lambda_{k}^{m_{k}} h_{m_{1}, \ldots, m_{k}}^{*}\left(P_{1}, \ldots, P_{k} ; \cdot\right)
\end{gathered}
$$

where each function $h_{m_{1}, \ldots, m_{k}}^{*}\left(P_{1}, \ldots, P_{k} ; \cdot\right)$ is a support function. For the proof of the latter fact, we use representations of the functions $h_{m_{1}, \ldots, m_{k}}^{*}\left(P_{1}, \ldots, P_{k} ; \cdot\right)$ which are new even in the case $k=2$ treated by Goodey and Weil. These authors obtained the convexity of the functions $h_{j}^{*}(K, M, \cdot)$ in an indirect way, constructing the first surface area measure of the putative polytope and then using an existence theorem. The extension 
to more than two polytopes by their method, if possible, would certainly be rather more cumbersome. We continue with a brief investigation of the mixed polytopes represented by the support functions $h_{m_{1}, \ldots, m_{k}}^{*}$; in particular, we give explicit formulae for their vertices and determine their edges.

We restrict ourselves here to polytopes and refer to [16] and [3] for the continuity and approximation arguments that permit an extension of the expansions from polytopes to general convex bodies.

In Section 6 we prove a general inclusion inequality for translation mixtures of convex bodies. A special case says that the body $T(K, K)$ contains a homothet of $K$ with homothety factor $(n+1)^{-1} V_{n}(K-K)$. The equality case characterizes simplices.

\section{Polytope Bundles}

First we wish to point out the analogy of the integral (1) to the definition of the fibre polytopes as introduced by Billera and Sturmfels [1]. The convex body $T(K, M)$ with support function (1) is a translate of the convex body $\tilde{T}(K, M)$ with support function

$$
\int_{\mathbb{R}^{n}} h(K \cap(M+x), \cdot) d x,
$$

where $h$ is the ordinary support function. Now let $K, M$ be polytopes, and let $Q:=\{x \in$ $\left.\mathbb{R}^{n}: K \cap(M+x) \neq \emptyset\right\}$; then $Q$ is a polytope. If $\mathcal{B}_{x}:=K \cap(M+x)$ for $x \in Q$, then $\mathcal{B}$ is a polytope bundle in the sense of Billera and Sturmfels [1], and $\tilde{T}(K, M)=\int_{Q} \mathcal{B}$ is the Minkowski integral of this polytope bundle. In contrast to this, Billera and Sturmfels considered projections $\pi: P \rightarrow Q$ between polytopes $P$ and $Q$ and defined the fibre polytope of $P$ over $Q$, up to a normalization, as the Minkowski integral $\int_{Q} \mathcal{B}$, where $\mathcal{B}_{x}=\pi^{-1}(x)$. Thus, the Billera-Sturmfels construction averages the intersections of a polytope with the translates of a subspace, whereas the Goodey-Weil construction averages the intersections of a polytope with the translates of a second polytope.

Some of the observations from [1] apply also to the present case. Let $P_{1}, \ldots, P_{k} \subset \mathbb{R}^{n}$ be polytopes $(k \geq 2)$. Define

$$
Q:=\left\{\left(x_{1}, \ldots, x_{k-1}\right) \in\left(\mathbb{R}^{n}\right)^{k-1}:\left(P_{1}+x_{1}\right) \cap \cdots \cap\left(P_{k-1}+x_{k-1}\right) \cap P_{k} \neq \emptyset\right\},
$$

and let $f:\left(\mathbb{R}^{n}\right)^{k} \rightarrow\left(\mathbb{R}^{n}\right)^{k-1}$ be the linear map given by

$$
f\left(y_{1}, \ldots, y_{k}\right):=\left(y_{k}-y_{1}, \ldots, y_{k}-y_{k-1}\right) .
$$

Then $f\left(P_{1} \times \cdots \times P_{k}\right)=Q$, hence $Q$ is a polytope. For $X:=\left(x_{1}, \ldots, x_{k-1}\right) \in Q$, let

$$
\mathcal{B}_{X}:=\left(P_{1}+x_{1}\right) \cap \cdots \cap\left(P_{k-1}+x_{k-1}\right) \cap P_{k} .
$$

Then $\mathcal{B}$ is a polytope bundle over the base polytope $Q$. Its Minkowski integral, $\int_{Q} \mathcal{B}=$ : $\tilde{T}\left(P_{1}, \ldots, P_{k}\right)$, is the convex body with support function given by

$$
\int_{\mathbb{R}^{n}} \cdots \int_{\mathbb{R}^{n}} h\left(\left(P_{1}+x_{1}\right) \cap \cdots \cap\left(P_{k-1}+x_{k-1}\right) \cap P_{k}, \cdot\right) d x_{1} \cdots d x_{k-1} .
$$


Consider the polyhedral subdivision of $Q$ defined as the common refinement of all the images of faces of $P_{1} \times \cdots \times P_{k}$ under the linear map $f$. The polytope bundle $\mathcal{B}$ is piecewise linear (see [1] for the definition) with respect to this subdivision. Therefore, Theorem 1.3 of [1] implies that $\tilde{T}\left(P_{1}, \ldots, P_{k}\right)$ is a polytope. (For $k=2$, this was proved in a different way by Goodey and Weil [3].) Also the proof of Theorem 1.5 of [1] carries over, showing that there exist finitely many points $X^{(1)}, \ldots, X^{(m)} \in Q$, $X^{(i)}=\left(x_{1}^{(i)}, \ldots, x_{k-1}^{(i)}\right)$, and positive numbers $a_{1}, \ldots, a_{m}$ so that

$$
\tilde{T}\left(P_{1}, \ldots, P_{k-1}\right)=\sum_{i=1}^{m} a_{i}\left(\left(P_{1}+x_{1}^{(i)}\right) \cap \cdots \cap\left(P_{k-1}+x_{k-1}^{(i)}\right) \cap P_{k}\right) .
$$

\section{Preliminaries}

For the further investigation, we first collect some notation. We work in $n$-dimensional real Euclidean vector space $\mathbb{R}^{n}(n \geq 3)$, with scalar product $\langle\cdot, \cdot\rangle$ and induced norm $\|\cdot\|$. The Lebesgue measure on $\mathbb{R}^{n}$ is denoted by $\lambda_{n}$, and the spherical Lebesgue measure on the unit sphere $S^{n-1}:=\left\{x \in \mathbb{R}^{n}:\|x\|=1\right\}$ by $\sigma_{n-1}$. The number $\kappa_{n}=\pi^{n / 2} / \Gamma(1+n / 2)$ is the volume of the $n$-dimensional unit ball. For an $m$-dimensional convex body $K$ in $\mathbb{R}^{n}$, we denote by $V_{m}(K)$ its $m$-dimensional volume and by

$$
z_{m+1}(K):=\int_{K} x d \lambda_{m}(x)
$$

its moment vector (see p. 303 of [8]; generally, we refer to [8] for terminology or notions from the theory of convex bodies that are not explained here).

For a polyhedral set $P$ (a nonempty intersection of finitely many closed halfspaces in $\mathbb{R}^{n}$ ) and a face $F$ of $P$, the normal cone of $P$ at $F$ is denoted by $N(P, F)$ and the (normalized) exterior angle of $P$ at $F$ by $\gamma(F, P)$. If $P_{1}, \ldots, P_{k}$ are polyhedral sets and $F_{i}$ is a face of $P_{i}$ for $i=1, \ldots, k$, then the common exterior angle $\gamma\left(F_{1}, \ldots, F_{k} ; P_{1}, \ldots, P_{k}\right)$ is defined by

$$
\gamma\left(F_{1}, \ldots, F_{k} ; P_{1}, \ldots, P_{k}\right):=\gamma\left(\bigcap_{i=1}^{k}\left(F_{i}-x_{i}\right), \bigcap_{i=1}^{k}\left(P_{i}-x_{i}\right)\right),
$$

where $x_{i} \in$ relint $F_{i}$ for $i=1, \ldots, k$ (the definition does not depend on the choice of the $x_{i}$ ). The corresponding normal cones satisfy

$$
N\left(\bigcap_{i=1}^{k}\left(P_{i}-x_{i}\right), \bigcap_{i=1}^{k}\left(F_{i}-x_{i}\right)\right)=N\left(P_{1}, F_{1}\right)+\cdots+N\left(P_{k}, F_{k}\right)
$$

(see [8], especially Sections 2.2 and 2.4).

Particular polyhedral sets which will play an essential role are the halfspaces

$$
H_{u, t}^{+}:=\left\{x \in \mathbb{R}^{n}:\langle x, u\rangle \geq t\right\},
$$

where $u \in S^{n-1}$ and $t \in \mathbb{R}$. The face

$$
H_{u, t}:=\left\{x \in \mathbb{R}^{n}:\langle x, u\rangle=t\right\}
$$


of $H_{u, t}^{+}$has the normal cone $N\left(H_{u, t}^{+}, H_{u, t}\right)=\operatorname{pos}\{-u\}:=\{-\lambda u: \lambda \geq 0\}$. We use the abbreviations $H_{u, 0}=: u^{\perp}$ and $H_{u, 0}^{+}=: u^{+}$. For a linear subspace $L$ of $\mathbb{R}^{n}, L^{\perp}$ is the orthogonal complement.

For a polyhedral set $P \subset \mathbb{R}^{n}$ and for $i=0, \ldots, n$, we denote by $\mathcal{F}_{i}(P)$ the set of $i$-dimensional faces of $P$. A 0 -dimensional face is of the form $\{e\}$, where $e$ is a vertex of $P$. In this case, we do not distinguish between $\{e\}$ and $e$, so that $\mathcal{F}_{0}(P)$ denotes also the set of vertices of $P$.

If $P$ is a polytope (a compact polyhedral set), then

$$
\sum_{e \in \mathcal{F}_{0}(P)} \gamma(e, P)=\chi(P)=1
$$

where $\chi$ denotes the Euler characteristic $(\chi(K)=1$ for a nonempty convex body $K$, and $\chi(\emptyset)=0)$. The sum

$$
\sum_{e \in \mathcal{F}_{0}(P)} \gamma(e, P) e=s(P)
$$

gives the Steiner point $s(P)$ of $P$, which for a convex body $K$ can also be represented by

$$
s(K)=\frac{1}{\kappa_{n}} \int_{S^{n-1}} h(K, u) u d \sigma_{n-1}(u)
$$

(see pp. 42 and 305 of [8]). Here, $h(K, \cdot)$ denotes the support function of $K$, defined by $h(K, u)=\max \{\langle x, u\rangle: x \in K\}$, for $u \in \mathbb{R}^{n}$. This definition is extended by $h(\emptyset, \cdot):=0$. The centred support function of $K$, denoted by $h^{*}(K, \cdot)$, is the support function of the translate of $K$ with the Steiner point at the origin, thus

$$
h^{*}(K, \cdot)=h(K, \cdot)-\langle s(K), \cdot\rangle .
$$

We recall (e.g., from [17]) the definition of the determinant of subspaces. Let $L_{1}, \ldots$, $L_{k}$ be linear subspaces of $\mathbb{R}^{n}$ with $\sum_{i=1}^{k} \operatorname{dim} L_{i}=m \leq n$. Choose an orthonormal basis in each $L_{i}$ (the empty set if $\operatorname{dim} L_{i}=0$ ) and let $\operatorname{det}\left(L_{1}, \ldots, L_{k}\right)$ be the $m$-dimensional volume of the parallelepiped spanned by the union of these bases (1, by definition, if $\operatorname{dim} L_{i}=0$ for $\left.i=1, \ldots, k\right)$. Then one defines

$$
\left[L_{1}, \ldots, L_{k}\right]:=\operatorname{det}\left(L_{1}^{\perp}, \ldots, L_{k}^{\perp}\right) \quad \text { if } \quad \sum_{i=1}^{k} \operatorname{dim} L_{i} \geq(k-1) n
$$

and $\left[L_{1}, \ldots, L_{k}\right]:=0$ if $\sum_{i=1}^{k} \operatorname{dim} L_{i}<(k-1) n$. Note that any $n$-dimensional argument of $\left[L_{1}, \ldots, L_{k}\right]$ can be deleted without changing the value, that $[L]=1$, and that $\left[L_{1}, \ldots, L_{k}\right]=0$ if $L_{1}, \ldots, L_{k}$ are not in general relative position.

For nonempty subsets $F_{1}, \ldots, F_{k} \subset \mathbb{R}^{n}$ we define $\left[F_{1}, \ldots, F_{k}\right]:=\left[L\left(F_{1}\right), \ldots\right.$, $L\left(F_{k}\right)$ ], where $L\left(F_{i}\right)$ is the linear subspace parallel to the affine hull of $F_{i}(i=1, \ldots, k)$.

Finally, $\mathbf{1}$ always denotes an indicator function. We use it in both versions: $\mathbf{1}_{A}(x)=1$ if $x$ is in the set $A$, or $\mathbf{1}\{E\}=1$ if $E$ is true, and $=0$ otherwise in either case. 


\section{Integral Geometry of Support Functions}

In the following, $P, P_{1}, \ldots, P_{k}$ are $n$-dimensional convex polytopes in $\mathbb{R}^{n}$. Unless stated otherwise, $u \in S^{n-1}$ is a given unit vector.

We start with the formula

$$
h^{*}(P, u)=\sum_{F \in \mathcal{F}_{1}(P)} \gamma\left(F, u^{\perp} ; P, u^{+}\right)\left[F, u^{\perp}\right] V_{1}(F),
$$

due to Weil [16, Corollary 2], for which we give a new and more direct proof. If $\alpha \in \mathbb{R}$ is a number satisfying $P \subset H_{u, \alpha}^{+}$, then

$$
\int_{\alpha}^{\infty} \chi\left(P \cap H_{u, t}^{+}\right) d t=\int_{\alpha}^{h(P, u)} 1 d t
$$

hence

$$
h(P, u)=\alpha+\int_{\alpha}^{\infty} \chi\left(P \cap H_{u, t}^{+}\right) d t
$$

Now, using (3),

$$
\begin{aligned}
\int_{\alpha}^{\infty} \chi\left(P \cap H_{u, t}^{+}\right) d t= & \int_{\alpha}^{\infty} \sum_{e \in \mathcal{F}_{0}\left(P \cap H_{u, t}^{+}\right)} \gamma\left(e, P \cap H_{u, t}^{+}\right) d t \\
= & \int_{\alpha}^{\infty} \sum_{e \in \mathcal{F}_{0}(P)} \gamma(e, P) \mathbf{1}\{\langle e, u\rangle \geq t\} d t \\
& +\int_{\alpha}^{\infty} \sum_{F \in \mathcal{F}_{1}(P)} \gamma\left(F, u^{\perp} ; P, u^{+}\right) \chi\left(F \cap H_{u, t}\right) d t \\
= & \sum_{e \in \mathcal{F}_{0}(P)} \gamma(e, P)(\langle e, u\rangle-\alpha) \\
& +\sum_{F \in \mathcal{F}_{1}(P)} \gamma\left(F, u^{\perp} ; P, u^{+}\right)\left[F, u^{\perp}\right] V_{1}(F) .
\end{aligned}
$$

By (3) and (4), the first summand is equal to $\langle s(P), u\rangle-\alpha$, hence (6) follows.

The following proposition extends (1) in the case of polytopes.

Proposition 4.1. If $P_{1}, \ldots, P_{k} \subset \mathbb{R}^{n}$ are polytopes $(k \geq 2)$, then

$$
\begin{gathered}
\int_{\mathbb{R}^{n}} \cdots \int_{\mathbb{R}^{n}} h^{*}\left(\left(P_{1}+x_{1}\right) \cap \cdots \cap\left(P_{k-1}+x_{k-1}\right) \cap P_{k}, \cdot\right) d x_{1} \cdots d x_{k-1} \\
=\sum_{\substack{m_{1}, \ldots, m_{k}=0 \\
m_{1}+\cdots+m_{k}=(k-1) n+1}}^{n} h_{m_{1}, \ldots, m_{k}}^{*}\left(P_{1}, \ldots, P_{k} ; \cdot\right)
\end{gathered}
$$

with

$$
h_{m_{1}, \ldots, m_{k}}^{*}\left(P_{1}, \ldots, P_{k} ; u\right)
$$




$$
\begin{aligned}
= & \sum_{F_{1} \in \mathcal{F}_{m_{1}}\left(P_{1}\right)} \ldots \sum_{F_{k} \in \mathcal{F}_{m_{k}}\left(P_{k}\right)} \gamma\left(F_{1}, \ldots, F_{k}, u^{\perp} ; P_{1}, \ldots, P_{k}, u^{+}\right) \\
& \times\left[F_{1}, \ldots, F_{k}, u^{\perp}\right] V_{m_{1}}\left(F_{1}\right) \cdots V_{m_{k}}\left(F_{k}\right)
\end{aligned}
$$

for $u \in S^{n-1}$.

This follows from a more general result of Weil [13]. In fact, relation (6) can be written in the form

$$
h^{*}(P, u)=\Phi_{1, n-1}^{(0)}\left(P, u^{+}, \mathbb{R}^{n} \times \beta(u)\right),
$$

where $\beta(u) \subset u^{\perp}$ is a Borel set with $(n-1)$-dimensional Lebesgue measure equal to 1. Here $\Phi_{1, n-1}^{(0)}$ is a mixed measure, see Definition (2) in [13]. Proposition 4.1 is now obtained by repeated applications of Corollary 3.5 in [13]. A short direct proof can also be given along the lines of the proof of Theorem 3.1 in [13], where the assumption of general position is not necessary. Theorem 4.2 to be proved below is more general than Proposition 4.1.

Once relation (8) has been proved, it is easy to see that each of the functions $h_{m_{1}, \ldots, m_{k}}^{*}\left(P_{1}, \ldots, P_{k} ; \cdot\right)$ is centred, that is, satisfies

$$
\int_{S^{n-1}} h_{m_{1}, \ldots, m_{k}}^{*}\left(P_{1}, \ldots, P_{k} ; u\right) u d \sigma_{n-1}(u)=0
$$

This follows after multiplying (8) (evaluated at $u$ ) by $u$, integrating over all $u \in S^{n-1}$ with respect to $\sigma_{n-1}$, and applying Fubini's theorem. Since $h^{*}(K, \cdot)$ is centred for every convex body $K$, this gives

$$
\sum_{m_{1}, \ldots, m_{k}} \int_{S^{n-1}} h_{m_{1}, \ldots, m_{k}}^{*}\left(P_{1}, \ldots, P_{k} ; u\right) u d \sigma_{n-1}(u)=0,
$$

and since the summands have different degrees of homogeneity in $P_{1}, \ldots, P_{k}$, each of them must vanish.

In (1), the right-hand side was written in a particular form. To obtain this, we note that (9) and (6) give

$$
\begin{aligned}
h_{1, n, \ldots, n}^{*}\left(P_{1}, \ldots, P_{k} ; u\right) & =\sum_{F_{1} \in \mathcal{F}_{1}\left(P_{1}\right)} \gamma\left(F_{1}, u^{\perp} ; P_{1}, u^{+}\right)\left[F_{1}, u^{\perp}\right] V_{1}\left(F_{1}\right) V_{n}\left(P_{2}\right) \cdots V_{n}\left(P_{k}\right) \\
& =V_{n}\left(P_{2}\right) \cdots V_{n}\left(P_{k}\right) h^{*}\left(P_{1}, u\right) .
\end{aligned}
$$

We turn now to the main part of this investigation, a class of representations for the functions $h_{m_{1}, \ldots, m_{k}}^{*}$, which are new even for $k=2$. They will, in particular, be useful in giving a simple proof of the fact that these functions are support functions.

We employ a special representation of the integral

$$
\int_{\mathbb{R}^{n}} \cdots \int_{\mathbb{R}^{n}} \chi\left(\left(P_{1}+x_{1}\right) \cap \cdots \cap\left(P_{k-1}+x_{k-1}\right) \cap P_{k}\right) d x_{1} \cdots d x_{k-1},
$$

which was obtained in [9]. 
Definition. The vector $w \in \mathbb{R}^{n}$ is admissible for $\left(P_{1}, \ldots, P_{k}\right)$ if

$$
w \notin N\left(P_{1}, F_{1}\right)+\cdots+N\left(P_{k}, F_{k}\right)
$$

whenever $F_{1} \in \mathcal{F}_{m_{1}}\left(P_{1}\right), \ldots, F_{k} \in \mathcal{F}_{m_{k}}\left(P_{k}\right)$ with numbers $m_{1}, \ldots, m_{k} \in\{1, \ldots, n\}$ satisfying $m_{1}+\cdots+m_{k}=(k-1) n+1$.

Admissibility implies the condition on $w$ as formulated in Theorem 2 of [9]. That theorem concerns the $(k-1) n$-dimensional volume $V_{(k-1) n}\left(D\left(P_{1}, \ldots, P_{k}\right)\right)$ of the set

$$
\begin{aligned}
& D\left(P_{1}, \ldots, P_{k}\right) \\
& \quad:=\left\{\left(x_{1}, \ldots, x_{k-1}\right) \in\left(\mathbb{R}^{n}\right)^{k-1}:\left(P_{1}+x_{1}\right) \cap \cdots \cap\left(P_{k-1}+x_{k-1}\right) \cap P_{k} \neq \emptyset\right\} .
\end{aligned}
$$

If $w$ is admissible for $\left(P_{1}, \ldots, P_{k}\right)$, then Theorem 2 of [9] gives

$$
\begin{aligned}
V_{(k-1) n}\left(D\left(P_{1}, \ldots, P_{k}\right)\right) \\
=\int_{\mathbb{R}^{n}} \cdots \int_{\mathbb{R}^{n}} \chi\left(\left(P_{1}+x_{1}\right) \cap \cdots \cap\left(P_{k-1}+x_{k-1}\right) \cap P_{k}\right) d x_{1} \cdots d x_{k-1} \\
=\sum_{\substack{m_{1}, \ldots, m_{k}=0 \\
m_{1}+\cdots+m_{k}=(k-1) n}}^{n} \sum_{F_{1} \in \mathcal{F}_{m_{1}}\left(P_{1}\right)} \cdots \sum_{F_{k} \in \mathcal{F}_{m_{k}}\left(P_{k}\right)} \mathbf{1}_{N\left(P_{1}, F_{1}\right)+\cdots+N\left(P_{k}, F_{k}\right)}(w) \\
\quad \times\left[F_{1}, \ldots, F_{k}\right] V_{m_{1}}\left(F_{1}\right) \cdots V_{m_{k}}\left(F_{k}\right) .
\end{aligned}
$$

Now we consider, first, the ordinary support function. Let polytopes $P_{1}, \ldots, P_{k} \subset \mathbb{R}^{n}$ and a unit vector $u \in S^{n-1}$ be given. We want to apply (11) with $P_{k}$ replaced by any nonempty intersection $P_{k} \cap H_{u, t}^{+}, t \in \mathbb{R}$.

Definition. The vector $w \in \mathbb{R}^{n}$ is admissible for $\left(P_{1}, \ldots, P_{k}, u\right)$ if it is admissible for $\left(P_{1}, \ldots, P_{k}\right)$ and if

$$
w \notin N\left(P_{1}, F_{1}\right)+\cdots+N\left(P_{k}, F_{k}\right)+\operatorname{pos}\{-u\}
$$

whenever $F_{1} \in \mathcal{F}_{m_{1}}\left(P_{1}\right), \ldots, F_{k} \in \mathcal{F}_{m_{k}}\left(P_{k}\right)$ with numbers $m_{1}, \ldots, m_{k} \in\{1, \ldots, n\}$ satisfying $m_{1}+\cdots+m_{k}=(k-1) n+2$.

If $w$ is admissible for $\left(P_{1}, \ldots, P_{k}, u\right)$, then $w$ is admissible for $\left(P_{1}, \ldots, P_{k-1}, P_{k} \cap H_{u, t}^{+}\right)$ whenever $P_{k} \cap H_{u, t}^{+}$is not empty. The vectors not admissible for $\left(P_{1}, \ldots, P_{k}, u\right)$ are contained in the union of finitely many proper linear subspaces of $\mathbb{R}^{n}$.

Let $w$ be admissible for $\left(P_{1}, \ldots, P_{k}, u\right)$. Choose a number $\alpha \in \mathbb{R}$ such that $P_{1}, \ldots$, $P_{k} \subset H_{u, \alpha}^{+}$. Using (7) and (11) (and recalling that $h(\emptyset, u)=0$ ), we get

$$
\begin{aligned}
I\left(P_{1}, \ldots,\right. & \left.P_{k}, u\right) \\
& :=\int_{\mathbb{R}^{n}} \cdots \int_{\mathbb{R}^{n}} h\left(\left(P_{1}+x_{1}\right) \cap \cdots \cap\left(P_{k-1}+x_{k-1}\right) \cap P_{k}, u\right) d x_{1} \cdots d x_{k-1} \\
= & \int_{D\left(P_{1}, \ldots, P_{k}\right)}\left[\alpha+\int_{\alpha}^{\infty} \chi\left(\left(P_{1}+x_{1}\right) \cap \cdots \cap\left(P_{k-1}+x_{k-1}\right) \cap P_{k} \cap H_{u, t}^{+}\right) d t\right] \\
& \times d x_{1} \cdots d x_{k-1}
\end{aligned}
$$




$$
\begin{aligned}
= & \alpha V_{(k-1) n}\left(D\left(P_{1}, \ldots, P_{k}\right)\right) \\
& +\int_{\alpha}^{\infty} \int_{\mathbb{R}^{n}} \ldots \int_{\mathbb{R}^{n}} \chi\left(\left(P_{1}+x_{1}\right) \cap \cdots\right. \\
& \left.\cap\left(P_{k-1}+x_{k-1}\right) \cap P_{k} \cap H_{u, t}^{+}\right) d x_{1} \cdots d x_{k-1} d t \\
& \alpha V_{(k-1) n}\left(D\left(P_{1}, \ldots, P_{k}\right)\right) \\
& +\int_{\alpha}^{\infty} \sum_{m_{m_{1}+\cdots+m_{k}=(k-1) n}}^{n} \sum_{F_{1} \in \mathcal{F}_{m_{1}}\left(P_{1}\right)} \ldots \\
& \sum_{F_{k-1} \in \mathcal{F}_{m_{k-1}}\left(P_{k-1}\right)} \sum_{F_{k} \in \mathcal{F}_{m_{k}}\left(P_{k} \cap H_{u, t}^{+}\right)} \mathbf{1}_{N\left(P_{1}, F_{1}\right)+\cdots+N\left(P_{k-1}, F_{k-1}\right)+N\left(P_{k} \cap H_{u, t}^{+}, F_{k}\right)}(w) \\
& \times\left[F_{1}, \ldots, F_{k}\right] V_{m_{1}}\left(F_{1}\right) \cdots V_{m_{k}}\left(F_{k}\right) d t .
\end{aligned}
$$

The $m_{k}$-faces $F_{k}$ of $P_{k} \cap H_{u, t}^{+}$(to the extent that they are relevant for the integration) are of two types, either

$$
F_{k}=F \cap H_{u, t}^{+} \quad \text { with } \quad F \in \mathcal{F}_{m_{k}}\left(P_{k}\right)
$$

or

$$
F_{k}=F \cap H_{u, t} \quad \text { with } \quad F \in \mathcal{F}_{m_{k}+1}\left(P_{k}\right) .
$$

Therefore, we have

$$
I\left(P_{1}, \ldots, P_{k}, u\right)=\alpha V_{(k-1) n}\left(D\left(P_{1}, \ldots, P_{k}\right)\right)+S_{1}+S_{2}
$$

with

$$
\begin{aligned}
S_{1}:= & \sum_{\substack{m_{1}, \ldots, m_{k}=0 \\
m_{1}+\cdots+m_{k}=(k-1) n}}^{n} \sum_{F_{1} \in \mathcal{F}_{m_{1}}\left(P_{1}\right)} \ldots \\
& \sum_{F_{k-1} \in \mathcal{F}_{m_{k-1}}\left(P_{k-1}\right)} \sum_{F \in \mathcal{F}_{m_{k}}\left(P_{k}\right)} I_{1}\left(m_{1}, \ldots, m_{k}, F_{1}, \ldots, F_{k-1}, F\right),
\end{aligned}
$$

where

$$
\begin{aligned}
I_{1}\left(m_{1}, \ldots, m_{k},\right. & \left.F_{1}, \ldots, F_{k-1}, F\right) \\
:= & \int_{\alpha}^{\infty} \mathbf{1}_{N\left(P_{1}, F_{1}\right)+\cdots+N\left(P_{k-1}, F_{k-1}\right)+N\left(P_{k} \cap H_{u, t}^{+}, F \cap H_{u, t}^{+}\right)}(w) \\
& \times\left[F_{1}, \ldots, F_{k-1}, F \cap H_{u, t}^{+}\right] V_{m_{1}}\left(F_{1}\right) \cdots V_{m_{k-1}}\left(F_{k-1}\right) V_{m_{k}}\left(F \cap H_{u, t}^{+}\right) d t,
\end{aligned}
$$

and

$$
\begin{aligned}
S_{2}:= & \sum_{\substack{m_{1}, \ldots, m_{k}=0 \\
m_{1}+\cdots+m_{k}=(k-1) n}}^{n} \sum_{F_{1} \in \mathcal{F}_{m_{1}}\left(P_{1}\right)} \ldots \\
& \sum_{F_{k-1} \in \mathcal{F}_{m_{k-1}}\left(P_{k-1}\right)} \sum_{F \in \mathcal{F}_{m_{k}+1}\left(P_{k}\right)} I_{2}\left(m_{1}, \ldots, m_{k}, F_{1}, \ldots, F_{k-1}, F\right)
\end{aligned}
$$


(with $\mathcal{F}_{n+1}\left(P_{k}\right)=\emptyset$ ), where

$$
\begin{aligned}
I_{2}\left(m_{1}, \ldots,\right. & \left.m_{k}, F_{1}, \ldots, F_{k-1}, F\right) \\
:= & \int_{\alpha}^{\infty} \mathbf{1}_{N\left(P_{1}, F_{1}\right)+\cdots+N\left(P_{k-1}, F_{k-1}\right)+N\left(P_{k} \cap H_{u, t}^{+}, F \cap H_{u, t}\right)}(w) \\
& \times\left[F_{1}, \ldots, F_{k-1}, F \cap H_{u, t}\right] V_{m_{1}}\left(F_{1}\right) \cdots V_{m_{k-1}}\left(F_{k-1}\right) V_{m_{k}}\left(F \cap H_{u, t}\right) d t .
\end{aligned}
$$

For the computation of $I_{1}\left(m_{1}, \ldots, m_{k}, F_{1}, \ldots, F_{k-1}, F\right)$, we need only consider those numbers $t \in \mathbb{R}$ for which $\operatorname{dim}\left(F \cap H_{u, t}^{+}\right)=m_{k}$. For these $t$, we have

$$
N\left(P_{k} \cap H_{u, t}^{+}, F \cap H_{u, t}^{+}\right)=N\left(P_{k}, F\right)
$$

and

$$
\left[F_{1}, \ldots, F_{k-1}, F \cap H_{u, t}^{+}\right]=\left[F_{1}, \ldots, F_{k-1}, F\right] .
$$

Further,

$$
\begin{aligned}
\int_{\alpha}^{\infty} V_{m_{k}}\left(F \cap H_{u, t}^{+}\right) d t & =\int_{\alpha}^{\infty} \int_{F} \mathbf{1}\{\langle y, u\rangle \geq t\} d \lambda_{m_{k}}(y) d t \\
& =\int_{F} \int_{\alpha}^{\langle y, u\rangle} d t d \lambda_{m_{k}}(y)=\int_{F}(\langle y, u\rangle-\alpha) d \lambda_{m_{k}}(y) \\
& =\left\langle z_{m_{k}+1}(F), u\right\rangle-\alpha V_{m_{k}}(F),
\end{aligned}
$$

with the moment vector $z_{m_{k}+1}(F)$ as defined by (2). Thus, we obtain

$$
\begin{aligned}
S_{1}= & \sum_{\substack{m_{1}, \ldots, m_{k}=0 \\
m_{1}+\cdots+m_{k}=(k-1) n}}^{n} \sum_{F_{1} \in \mathcal{F}_{m_{1}}\left(P_{1}\right)} \ldots \sum_{F_{k} \in \mathcal{F}_{m_{k}}\left(P_{k}\right)} \mathbf{1}_{N\left(P_{1}, F_{1}\right)+\cdots+N\left(P_{k}, F_{k}\right)}(w) \\
& \times\left[F_{1}, \ldots, F_{k}\right] V_{m_{1}}\left(F_{1}\right) \cdots V_{m_{k-1}}\left(F_{k-1}\right)\left(\left\langle z_{m_{k}+1}\left(F_{k}\right), u\right\rangle-\alpha V_{m_{k}}\left(F_{k}\right)\right) \\
= & -\alpha V_{(k-1) n}\left(D\left(P_{1}, \ldots, P_{k}\right)\right) \\
& +\sum_{\substack{m_{1} \ldots, \ldots m_{k}=0 \\
m_{1}+\cdots+m_{k}=(k-1) n}} \sum_{F_{1} \in \mathcal{F}_{m_{1}}\left(P_{1}\right)} \cdots \sum_{F_{k} \in \mathcal{F}_{m_{k}}\left(P_{k}\right)} \mathbf{1}_{N\left(P_{1}, F_{1}\right)+\cdots+N\left(P_{k}, F_{k}\right)}(w) \\
& \times\left[F_{1}, \ldots, F_{k}\right] V_{m_{1}}\left(F_{1}\right) \cdots V_{m_{k-1}}\left(F_{k-1}\right)\left\langle z_{m_{k}+1}\left(F_{k}\right), u\right\rangle,
\end{aligned}
$$

with the help of (11).

For the computation of $I_{2}\left(m_{1}, \ldots, m_{k}, F_{1}, \ldots, F_{k-1}, F\right)$, we need only consider those numbers $t \in \mathbb{R}$ for which $\operatorname{dim}\left(F \cap H_{u, t}\right)=m_{k}$. For these $t$, we have

$$
N\left(P_{k} \cap H_{u, t}^{+}, F \cap H_{u, t}\right)=N\left(P_{k}, F\right)+\operatorname{pos}\{-u\} ;
$$

further

$$
\int_{\alpha}^{\infty} V_{m_{k}}\left(F \cap H_{u, t}\right) d t=\left[F, u^{\perp}\right] V_{m_{k}+1}(F)
$$


and

$$
\left[F_{1}, \ldots, F_{k-1}, F \cap H_{u, t}\right]\left[F, u^{\perp}\right]=\left[F_{1}, \ldots, F_{k-1}, F, u^{\perp}\right]
$$

(see Proposition 2.1 in [17]). Thus, we get

$$
\begin{aligned}
S_{2}= & \sum_{\substack{m_{1}, \ldots, m_{k}=0 \\
m_{1}+\cdots+m_{k}=(k-1) n}}^{n} \sum_{F_{1} \in \mathcal{F}_{m_{1}}\left(P_{1}\right)} \ldots \\
& \sum \sum_{F_{k-1} \in \mathcal{F}_{m_{k-1}}\left(P_{k-1}\right)} \sum_{F_{k} \in \mathcal{F}_{m_{k}+1}\left(P_{k}\right)} \mathbf{1}_{N\left(P_{1}, F_{1}\right)+\cdots+N\left(P_{k}, F_{k}\right)+\operatorname{pos}\{-u\}}(w)\left[F_{1}, \ldots, F_{k}, u^{\perp}\right] \\
& \times V_{m_{1}}\left(F_{1}\right) \cdots V_{m_{k-1}}\left(F_{k-1}\right) V_{m_{k}+1}\left(F_{k}\right) .
\end{aligned}
$$

We have obtained the following result.

Theorem 4.2. If $P_{1}, \ldots, P_{k} \subset \mathbb{R}^{n}$ are polytopes $(k \geq 2), u \in S^{n-1}$, and the vector $w$ is admissible for $\left(P_{1}, \ldots, P_{k}, u\right)$, then

$$
\begin{aligned}
& \int_{\mathbb{R}^{n}} \cdots \int_{\mathbb{R}^{n}} h\left(\left(P_{1}+x_{1}\right) \cap \cdots \cap\left(P_{k-1}+x_{k-1}\right) \cap P_{k}, u\right) d x_{1} \cdots d x_{k-1} \\
&=\sum_{\substack{m_{1}, \ldots, m_{k}=0 \\
m_{1}+\cdots+m_{k}=(k-1) n+1}}^{n} \sum_{F_{1} \in \mathcal{F}_{m_{1}}\left(P_{1}\right)} \cdots \\
& \sum_{F_{k-1} \in \mathcal{F}_{m_{k-1}}\left(P_{k-1}\right)} \sum_{F_{k} \in \mathcal{F}_{m_{k}-1}\left(P_{k}\right)} \mathbf{1}_{N\left(P_{1}, F_{1}\right)+\cdots+N\left(P_{k}, F_{k}\right)}(w) \\
& \times\left[F_{1}, \ldots, F_{k}\right] V_{m_{1}}\left(F_{1}\right) \cdots V_{m_{k-1}}\left(F_{k-1}\right)\left\langle z_{m_{k}}\left(F_{k}\right), u\right\rangle \\
&+\sum_{\substack{m_{1}, \ldots, m_{k}=0 \\
m_{1}+\cdots+m_{k}=(k-1) n+1}}^{n} \sum_{F_{1} \in \mathcal{F}_{m_{1}}\left(P_{1}\right)} \cdots \sum_{F_{k} \in \mathcal{F}_{m_{k}}\left(P_{k}\right)} \mathbf{1}_{N\left(P_{1}, F_{1}\right)+\cdots+N\left(P_{k}, F_{k}\right)+\operatorname{pos}\{-u\}}(w) \\
& \times\left[F_{1}, \ldots, F_{k}, u^{\perp}\right] V_{m_{1}}\left(F_{1}\right) \cdots V_{m_{k}}\left(F_{k}\right) .
\end{aligned}
$$

We want to show that Theorem 4.2 is a more general result than Proposition 4.1, in so far as the latter can be derived from (12) by integration. For this, we need a translative intersection formula for the Steiner point.

Theorem 4.3. For polytopes $P_{1}, \ldots, P_{k} \subset \mathbb{R}^{n}(k \geq 2)$, the Steiner point satisfies the translative formula

$$
\begin{gathered}
\int_{\mathbb{R}^{n}} \cdots \int_{\mathbb{R}^{n}} s\left(\left(P_{1}+x_{1}\right) \cap \cdots \cap\left(P_{k-1}+x_{k-1}\right) \cap P_{k}\right) d x_{1} \cdots d x_{k-1} \\
=\sum_{\substack{m_{1}, \ldots, m_{k}=0 \\
m_{1}+\cdots+m_{k}=(k-1) n}}^{n} s_{m_{1}, \ldots, m_{k}}\left(P_{1}, \ldots, P_{k}\right)
\end{gathered}
$$


with

$$
\begin{aligned}
s_{m_{1}, \ldots, m_{k}}\left(P_{1}, \ldots, P_{k}\right)= & \sum_{F_{1} \in \mathcal{F}_{m_{1}}\left(P_{1}\right)} \ldots \sum_{F_{k} \in \mathcal{F}_{m_{k}}\left(P_{k}\right)} \gamma\left(F_{1}, \ldots, F_{k} ; P_{1}, \ldots, P_{k}\right) \\
& \times\left[F_{1}, \ldots, F_{k}\right] V_{m_{1}}\left(F_{1}\right) \cdots V_{m_{k-1}}\left(F_{k-1}\right) z_{m_{k}+1}\left(F_{k}\right) .
\end{aligned}
$$

This result can be deduced from the translative integral formula for curvature measures [13, Theorem 3.1 and (2)], since the Steiner point can be represented by the moment vector of the Gaussian curvature measure. For the reader's convenience, we give the short proof of this special case. Using (4), we get

$$
\begin{aligned}
\int_{\mathbb{R}^{n}} \cdots \int_{\mathbb{R}^{n}} s & \left(\left(P_{1}+x_{1}\right) \cap \cdots \cap\left(P_{k-1}+x_{k-1}\right) \cap P_{k}\right) d x_{1} \cdots d x_{k-1} \\
= & \int_{\mathbb{R}^{n}} \cdots \int_{\mathbb{R}^{n}} \sum_{\substack{e \in \mathcal{F}_{0}\left(\left(P_{1}+x_{1}\right) \cap \cdots \cap\left(P_{k-1}+x_{k-1}\right) \cap P_{k}\right) \\
\cap}} \gamma\left(e,\left(P_{1}+x_{1}\right) \cap \cdots\right. \\
= & \left.\left.\int_{\mathbb{R}^{n}} \cdots \int_{\mathbb{R}^{n}} \sum_{\substack{m_{1}, \ldots, m_{k}=0 \\
m_{1}+\cdots+m_{k}=(k-1) n}}^{n} \sum_{F_{1} \in \mathcal{F}_{m_{1}}\left(P_{1}\right)} \ldots \sum_{F_{k} \in \mathcal{F}_{m_{k}}\left(P_{k}\right)} \gamma\left(F_{k-1}\right) \cap P_{k}\right) e d x_{1} \cdots d x_{k-1}, F_{k} ; P_{1}, \ldots, P_{k}\right) \\
& \times s\left(\left(F_{1}+x_{1}\right) \cap \cdots \cap\left(F_{k-1} \cap x_{k-1}\right) \cap F_{k}\right) d x_{1} \cdots d x_{k-1} .
\end{aligned}
$$

For $F_{1} \in \mathcal{F}_{m_{1}}\left(P_{1}\right), \ldots, F_{k} \in \mathcal{F}_{m_{k}}\left(P_{k}\right)$ in general relative position, an appropriate splitting of the integration gives

$$
\begin{gathered}
\int_{\mathbb{R}^{n}} \cdots \int_{\mathbb{R}^{n}} s\left(\left(F_{1}+x_{1}\right) \cap \cdots \cap\left(F_{k-1}+x_{k-1}\right) \cap F_{k}\right) d x_{1} \cdots d x_{k-1} \\
=\left[F_{1}, \ldots, F_{k}\right] V_{m_{1}}\left(F_{1}\right) \cdots V_{m_{k-1}}\left(F_{k-1}\right) z_{m_{k}+1}\left(F_{k}\right),
\end{gathered}
$$

which completes the proof.

Formula (12) holds for all vectors $w \in S^{n-1}$ with the exception of those in a set of spherical Lebesgue measure zero. We integrate (12) over all $w$ with respect to the normalized spherical Lebesgue measure. On the right-hand side, the first multiple sum (where we rename $m_{k}$ as $m_{k}+1$ ) gives a contribution

$$
\begin{aligned}
\sum_{\substack{m_{1}, \ldots, m_{k}=0 \\
m_{1}+\cdots+m_{k}=(k-1) n}}^{n} & \sum_{F_{1} \in \mathcal{F}_{m_{1}}\left(P_{1}\right)} \ldots \sum_{F_{k} \in \mathcal{F}_{m_{k}}\left(P_{k}\right)} \gamma\left(F_{1}, \ldots, F_{k} ; P_{1}, \ldots, P_{k}\right) \\
& \times\left[F_{1}, \ldots, F_{k}\right] V_{m_{1}}\left(F_{1}\right) \cdots V_{m_{k-1}}\left(F_{k-1}\right)\left\langle z_{m_{k}+1}\left(F_{k}\right), u\right\rangle \\
= & \int_{\mathbb{R}^{n}} \cdots \int_{\mathbb{R}^{n}}\left\langle s\left(\left(P_{1}+x_{1}\right) \cap \cdots \cap\left(P_{k-1}+x_{k-1}\right) \cap P_{k}\right), u\right\rangle d x_{1} \cdots d x_{k-1},
\end{aligned}
$$

by (13), (14). The second multiple sum gives

$$
\begin{aligned}
\sum_{\substack{m_{1}, \ldots, m_{k}=0 \\
m_{1}+\cdots+m_{k}=(k-1) n+1}}^{n} & \sum_{F \in \mathcal{F}_{m_{1}}\left(P_{1}\right)} \ldots \sum_{F_{k} \in \mathcal{F}_{m_{k}}\left(P_{k}\right)} \gamma\left(F_{1}, \ldots, F_{k}, u^{\perp} ; P_{1}, \ldots, P_{k}, u^{+}\right) \\
& \times\left[F_{1}, \ldots, F_{k}, u^{\perp}\right] V_{m_{1}}\left(F_{1}\right) \cdots V_{m_{k}}\left(F_{k}\right)
\end{aligned}
$$




$$
=\sum_{\substack{m_{1}, \ldots, m_{k}=0 \\ m_{1}+\cdots+m_{k}=(k-1) n+1}}^{n} h_{m_{1}, \ldots, m_{k}}^{*}\left(P_{1}, \ldots, P_{k} ; u\right),
$$

where the latter functions are defined by (9). Proposition 4.1 follows by (5).

The advantage of Theorem 4.2 over Proposition 4.1 lies in its greater flexibility, due to the fact that the admissible vector $w$ is at our disposal. In particular, this enables us to give a short proof for the fact that the functions $h_{m_{1} \ldots, m_{k}}^{*}\left(P_{1}, \ldots, P_{k} ; \cdot\right)$ are support functions. For $k=2$, this was proved by Goodey and Weil [3], in a less direct way.

Theorem 4.4. If $P_{1}, \ldots, P_{k} \subset \mathbb{R}^{n}$ are polytopes, then the functions

$$
h_{m_{1}, \ldots, m_{k}}^{*}\left(P_{1}, \ldots, P_{k} ; \cdot\right)
$$

appearing in Proposition 4.1 are support functions.

Proof. We extend the functions $h_{m_{1}, \ldots, m_{k}}^{*}\left(P_{1}, \ldots, P_{k} ; \cdot\right)$ to $\mathbb{R}^{n}$ by positive homogeneity. Then we have to show that these extended functions are convex.

By (5), the integrals (8) and (12) differ only by a linear function. Comparing components of equal degrees of homogeneity in $P_{1}, \ldots, P_{k}$, we deduce from Proposition 4.1 and Theorem 4.2 (observing that the first multiple sum on the right-hand side of (12) is linear in $u$ ) that

$$
\begin{aligned}
h_{m_{1}, \ldots, m_{k}}^{*}\left(P_{1}, \ldots, P_{k} ; u\right) \\
=\left\langle v_{m_{1}, \ldots, m_{k}}\left(P_{1}, \ldots, P_{k}, w\right), u\right\rangle \\
\quad+\sum_{F_{1} \in \mathcal{F}_{m_{1}}\left(P_{1}\right)} \ldots \sum_{F_{k} \in \mathcal{F}_{m_{k}}\left(P_{k}\right)} \mathbf{1}_{N\left(P_{1}, F_{1}\right)+\cdots+N\left(P_{k}, F_{k}\right)+\operatorname{pos}\{-u\}}(w) \\
\quad \times\|u\|\left[F_{1}, \ldots, F_{k}, u^{\perp}\right] V_{m_{1}}\left(F_{1}\right) \cdots V_{m_{k}}\left(F_{k}\right)
\end{aligned}
$$

for $u \in \mathbb{R}^{n}$, if $w$ is admissible for $\left(P_{1}, \ldots, P_{k}, u\right)$. Here $v_{m_{1}, \ldots, m_{k}}\left(P_{1}, \ldots, P_{k}, w\right)$ is a certain vector, an explicit representation of which is given by (21) below, but is not needed in the present proof. The factor $\|u\|$ on the right-hand side of (15) appears because $h_{m_{1}, \ldots, m_{k}}^{*}\left(P_{1}, \ldots, P_{k} ; \cdot\right)$ is now positively homogeneous.

For given faces $F_{1} \in \mathcal{F}_{m_{1}}\left(P_{1}\right), \ldots, F_{k} \in \mathcal{F}_{m_{k}}\left(P_{k}\right)$ (where $m_{1}+\cdots+m_{k}=$ $(k-1) n+1)$, the definition of $\left[F_{1}, \ldots, F_{k}, u^{\perp}\right]$ entails that

$$
f_{F_{1}, \ldots, F_{k}}(u):=\|u\|\left[F_{1}, \ldots, F_{k}, u^{\perp}\right]=\left|\operatorname{det}\left(e_{1}, \ldots, e_{n-1}, u\right)\right|,
$$

where the set $\left\{e_{1}, \ldots, e_{n-1}\right\}$ is composed of orthonormal bases of $L\left(F_{1}\right)^{\perp}, \ldots, L\left(F_{k}\right)^{\perp}$. Being the absolute value of a linear function, the function $f_{F_{1}, \ldots, F_{k}}$ is convex.

Let $u \in \mathbb{R}^{n} \backslash\{0\}$. Let $w \in \mathbb{R}^{n}$ be admissible for $\left(P_{1}, \ldots, P_{k}, u\right)$, thus

$$
w \notin N\left(P_{1}, F_{1}\right)+\cdots+N\left(P_{k}, F_{k}\right)
$$

if $F_{1} \in \mathcal{F}_{m_{1}}\left(P_{1}\right), \ldots, F_{k} \in \mathcal{F}_{m_{k}}\left(P_{k}\right), m_{1}+\cdots+m_{k}=(k-1) n+1$, and

$$
w \notin N\left(P_{1}, F_{1}\right)+\cdots+N\left(P_{k}, F_{k}\right)+\operatorname{pos}\{-u\}
$$


if $F_{1} \in \mathcal{F}_{m_{1}}\left(P_{1}\right), \ldots, F_{k} \in \mathcal{F}_{m_{k}}\left(P_{k}\right), m_{1}+\cdots+m_{k}=(k-1) n+2$. The righthand sides of (17) and (18) make up a set of finitely many closed convex cones, say $C_{1}(u), \ldots, C_{m}(u)$ (though not all of them depend on $u$ ), of dimensions less than $n$.

Now let $u_{0} \in \mathbb{R}^{n} \backslash\{0\}$ be given. Let $w \in \mathbb{R}^{n} \backslash \bigcup_{r=1}^{m} C_{r}\left(u_{0}\right)$. Then $w$ is admissible for $\left(P_{1}, \ldots, P_{k}, u_{0}\right)$. Each cone (18) depends continuously on $u$. Hence, there is a convex neighbourhood $U$ of $u_{0}$ in $\mathbb{R}^{n} \backslash\{0\}$ such that, for all $u \in U$, we have $w \notin \bigcup_{r=1}^{m} C_{r}(u)$, which means that $w$ is admissible for $\left(P_{1}, \ldots, P_{k}, u\right)$. Thus, the representation (15) holds for all $u \in U$ with the same vector $w$.

Let $F_{1} \in \mathcal{F}_{m_{1}}\left(P_{1}\right), \ldots, F_{k} \in \mathcal{F}_{m_{k}}\left(P_{k}\right)$, where $m_{1}+\cdots+m_{k}=(k-1) n+1$. The function

$$
u \mapsto \mathbf{1}_{N\left(P_{1}, F_{1}\right)+\cdots+N\left(P_{k}, F_{k}\right)+\operatorname{pos}\{-u\}}(w)
$$

is constant on $U$, since the boundary of the cone $N\left(P_{1}, F_{1}\right)+\cdots+N\left(P_{k}, F_{k}\right)+\operatorname{pos}\{-u\}$ is covered by the cones $C_{1}(u), \ldots, C_{m}(u)$, and $w \notin \bigcup_{r=1}^{m} C_{r}(u)$ for $u \in U$.

Therefore, on the cone pos $U$, the function $h_{m_{1}, \ldots, m_{k}}^{*}\left(P_{1}, \ldots, P_{k} ; \cdot\right)$ is the sum of a linear function and a linear combination, with constant positive coefficients, of convex functions. Thus, every point of $\mathbb{R}^{n} \backslash\{0\}$ has a neighbourhood on which $h_{m_{1}, \ldots, m_{k}}^{*}\left(P_{1}, \ldots, P_{k} ; \cdot\right)$ is convex. It follows that $h_{m_{1}, \ldots, m_{k}}^{*}\left(P_{1}, \ldots, P_{k} ; \cdot\right)$ is convex on $\mathbb{R}^{n}$.

The convex body with support function $h_{m_{1}, \ldots, m_{k}}^{*}\left(P_{1}, \ldots, P_{k} ; \cdot\right)$ is denoted by $T_{m_{1}, \ldots, m_{k}}$ $\left(P_{1}, \ldots, P_{k}\right)$. By Theorem 4.1, $T_{m_{1}, \ldots, m_{k}}\left(P_{1}, \ldots, P_{k}\right)$ is a summand of the translation mixture $T\left(P_{1}, \ldots, P_{k}\right)$, which by Section 2 is a polytope. Hence, the body $T_{m_{1}, \ldots, m_{k}}$ $\left(P_{1}, \ldots, P_{k}\right)$ is itself a polytope.

\section{Vertices and Edges of Mixed Polytopes}

Goodey and Weil [3] have determined the first surface area measures of the mixed polytopes $T_{m_{1}, m_{2}}\left(P_{1}, P_{2}\right)$. We extend their result to $k \geq 2$ polytopes, with a more direct proof, which in addition provides explicit representations for the vertices of $T_{m_{1}, \ldots, m_{k}}\left(P_{1}, \ldots, P_{k}\right)$.

Let $w \in \mathbb{R}^{n}$ be admissible for $\left(P_{1}, \ldots, P_{k}\right)$. Let $A_{w}$ be the union of the linear hulls

$$
\operatorname{lin}\left(\{w\} \cup N\left(P_{1}, F_{1}\right) \cup \cdots \cup N\left(P_{k}, F_{k}\right)\right),
$$

taken over all $F_{1} \in \mathcal{F}_{m_{1}}\left(P_{1}\right), \ldots, F_{k} \in \mathcal{F}_{m_{k}}\left(P_{k}\right)$ and all $m_{1}, \ldots, m_{k}$ with $m_{1}+\cdots+m_{k}=$ $(k-1) n+2$. Then $A_{w}$ is a union of finitely many linear subspaces of dimensions less than $n$. Let $u \in A_{w}^{c}:=\mathbb{R}^{n} \backslash A_{w}, u \neq 0$. Suppose that $w$ were not admissible for $\left(P_{1}, \ldots, P_{k}, u\right)$. Then there exist $m_{1}, \ldots, m_{k}$ with $m_{1}+\cdots+m_{k}=(k-1) n+2$ and faces $F_{1} \in \mathcal{F}_{m_{1}}\left(P_{1}\right), \ldots, F_{k} \in \mathcal{F}_{m_{k}}\left(P_{k}\right)$ such that

$$
w \in N\left(P_{1}, F_{1}\right)+\cdots+N\left(P_{k}, F_{k}\right)+\operatorname{pos}\{-u\} .
$$

Here $w \notin N\left(P_{1}, F_{1}\right)+\cdots+N\left(P_{k}, F_{k}\right)$, since otherwise $w \in N\left(P_{1}, F_{1}\right)+\cdots+$ $N\left(P_{r}, F_{r}^{\prime}\right)+\cdots+N\left(P_{k}, F_{k}\right)$ with suitable $r \in\{1, \ldots, k\}$ and $F_{r}^{\prime} \in \mathcal{F}_{m_{r}-1}\left(P_{r}\right)$, contradicting the assumption that $w$ is admissible for $\left(P_{1}, \ldots, P_{k}\right)$. Thus (19) implies

$$
u \in \operatorname{lin}\left(\{w\} \cup N\left(P_{1}, F_{1}\right) \cup \cdots \cup N\left(P_{k}, F_{k}\right)\right) \subset A_{w},
$$


a contradiction. This shows that $w$ is admissible for $\left(P_{1}, \ldots, P_{k}, u\right)$. Therefore, (15) holds for all $u \in A_{w}^{c}$.

Let $C_{1}, \ldots, C_{p}$ be the convex cones

$$
N\left(P_{1}, F_{1}\right)+\cdots+N\left(P_{k}, F_{k}\right),
$$

where $F_{1} \in \mathcal{F}_{m_{1}}\left(P_{1}\right), \ldots, F_{k} \in \mathcal{F}_{m_{k}}\left(P_{k}\right)$ and $m_{1}+\cdots+m_{k}=(k-1) n+1$. Then $w \notin C_{i}$ for $i=1, \ldots, p$, since $w$ is admissible for $\left(P_{1}, \ldots, P_{k}\right)$. Therefore, we can choose an $n$-dimensional closed convex cone $Z \subset \mathbb{R}^{n}$ with $w \in$ int $Z$ and such that

$$
C_{i} \cap \operatorname{int} Z=\emptyset \quad \text { for } \quad i=1, \ldots, p .
$$

Let $u \in Z \cap A_{w}^{c}$. Any cone $C_{i}$ can be separated from $Z$ by a hyperplane through 0 . Since $w \in$ int $Z$, it follows that $w \notin C_{i}+\operatorname{pos}\{-u\}$. Therefore, (15) gives

$$
h_{m_{1}, \ldots, m_{k}}^{*}\left(P_{1}, \ldots, P_{k} ; u\right)=\left\langle v_{m_{1}, \ldots, m_{k}}\left(P_{1}, \ldots, P_{k}, w\right), u\right\rangle
$$

for $u \in Z \cap A_{w}^{c}$. By continuity, (20) holds for all $u \in Z$. This shows that $v_{m_{1}, \ldots, m_{k}}\left(P_{1}, \ldots\right.$, $\left.P_{k}, w\right)$ is a vertex of $T_{m_{1}, \ldots, m_{k}}\left(P_{1}, \ldots, P_{k}\right)$, and $w$ is an exterior normal vector at this vertex.

The explicit formula for $v_{m_{1}, \ldots, m_{k}}\left(P_{1}, \ldots, P_{k}, w\right)$ is easily derived from Proposition 4.1 and Theorems 4.2 and 4.3, using also (5) and (15). In this way, we get the following result.

Theorem 5.1. Let $w$ be an admissible vector for $\left(P_{1}, \ldots, P_{k}\right)$. Let $m_{1}, \ldots, m_{k} \in$ $\{1, \ldots, n\}$ and $m_{1}+\cdots+m_{k}=(k-1) n+1$. Then the vertex of the polytope $T_{m_{1}, \ldots, m_{k}}\left(P_{1}, \ldots, P_{k}\right)$ with outer normal vector $w$ is unique and is given by

$$
\begin{aligned}
& v_{m_{1}, \ldots, m_{k}}\left(P_{1}, \ldots, P_{k}, w\right) \\
&=\sum_{F_{1} \in \mathcal{F}_{m_{1}}\left(P_{1}\right)} \ldots \sum_{F_{k-1} \in \mathcal{F}_{m_{k-1}}\left(P_{k-1}\right)} \\
& \times \sum_{F_{k} \in \mathcal{F}_{m_{k}-1}\left(P_{k}\right)}\left\{\mathbf{1}_{N\left(P_{1} F_{1}\right)+\cdots+N\left(P_{k}, F_{k}\right)}(w)-\gamma\left(F_{1}, \ldots, F_{k} ; P_{1}, \ldots, P_{k}\right)\right\} \\
& \times\left[F_{1}, \ldots, F_{k}\right] V_{m_{1}}\left(F_{1}\right) \cdots V_{m_{k-1}}\left(F_{k-1}\right) z_{m_{k}}\left(F_{k}\right) .
\end{aligned}
$$

We remark that integration of (21) over $w \in S^{n-1}$ with respect to the normalized spherical Lebesgue measure gives $s\left(T_{m_{1}, \ldots, m_{k}}\left(P_{1}, \ldots, P_{k}\right)\right)=0$; this is already known from (10).

Now we describe the first surface area measure (equivalently, the normal cones and lengths of the edges) of the polytope $T_{m_{1}, \ldots, m_{k}}\left(P_{1}, \ldots, P_{k}\right)$ for given $m_{1}, \ldots, m_{k}$ with $m_{1}+\cdots+m_{k}=(k-1) n+1$, but only under the following assumption of general position.

Assumption 5.2. If $F_{1} \in \mathcal{F}_{m_{1}}\left(P_{1}\right), \ldots, F_{k} \in \mathcal{F}_{m_{k}}\left(P_{k}\right)$, then the intersection

$$
L\left(F_{1}, \ldots, F_{k}\right):=L\left(F_{1}\right) \cap \cdots \cap L\left(F_{k}\right)
$$

of the linear subspaces parallel to the faces satisfies $\operatorname{dim} L\left(F_{1}, \ldots, F_{k}\right)=1$. All the onedimensional linear subspaces $L\left(F_{1}, \ldots, F_{k}\right)$ arising in this way are pairwise distinct. 
For each $i \in\{1, \ldots, p\}$, the closures of the connected components of $C_{i} \backslash \bigcup_{j \neq i} C_{j}$ are $(n-1)$-dimensional convex cones. Let $N_{1}, \ldots, N_{q}$ be the cones obtained in this way, for $i \in\{1, \ldots, p\}$. It follows from the previous considerations that the normal cones of the edges of $T_{m_{1}, \ldots, m_{k}}\left(P_{1}, \ldots, P_{k}\right)$ are among the cones $N_{1}, \ldots, N_{q}$. We show now that each $N_{i}$ does, in fact, belong to an edge of $T_{m_{1}, \ldots, m_{k}}\left(P_{1}, \ldots, P_{k}\right)$.

Let $N$ be one of the cones $N_{1}, \ldots, N_{q}$. It is contained in one of the cones $C_{1}, \ldots, C_{p}$, by Assumption 5.2 in a unique one, say $N \subset C_{1}$. Let $H^{+}, H^{-}$be the two closed halfspaces bounded by the linear hull of $N$. Let $x \in \operatorname{relint} N$. There are a convex neighbourhood $U$ of $x$ and vertices $v_{i}, v_{j}$ (not necessarily distinct, for the moment) of $T_{m_{1}, \ldots, m_{k}}\left(P_{1}, \ldots, P_{k}\right)$ with corresponding normal cones $Z_{i}, Z_{j}$ such that $U \cap H^{+} \subset Z_{i}$ and $U \cap H^{-} \subset Z_{j}$. We choose $U$ in such a way that $U \cap C_{i}=\emptyset$ for $i=2, \ldots, p$ and $U \cap \operatorname{lin} N \subset N$. Let $w \in U \cap$ int $Z_{i}$ be admissible for $\left(P_{1}, \ldots, P_{k}\right)$. Then (20) holds for $u \in U \cap Z_{i}$, thus $v_{i}=v_{m_{1}, \ldots, m_{k}}\left(P_{1}, \ldots, P_{k}, w\right)$. Let $u^{\prime} \in A_{w}^{c} \cap U \cap Z_{j}$. Then (15) holds for $u^{\prime}$. The same argument as used before Theorem 5.1 shows that $w \notin C_{r}+\operatorname{pos}\left\{-u^{\prime}\right\}$ for $r=2, \ldots, p$. On the other hand, the segment with endpoints $w$ and $u^{\prime}$ meets $C_{1}$, hence $w \in C_{1}+\operatorname{pos}\left\{-u^{\prime}\right\}$. Therefore, (15) gives

$$
\begin{aligned}
h_{m_{1}, \ldots, m_{k}}^{*}\left(P_{1}, \ldots, P_{k} ; u^{\prime}\right) & \\
& =\left\langle v_{m_{1}, \ldots, m_{k}}\left(P_{1}, \ldots, P_{k}, w\right), u^{\prime}\right\rangle+f_{F_{1}, \ldots, F_{k}}\left(u^{\prime}\right) V_{m_{1}}\left(F_{1}\right) \cdots V_{m_{k}}\left(F_{k}\right),
\end{aligned}
$$

where $F_{1}, \ldots, F_{k}$ are determined by $C_{1}=N\left(P_{1}, F_{1}\right)+\cdots+N\left(P_{k}, F_{k}\right)$. If $e_{1}, \ldots, e_{n-1}$ have the same meaning as in (16), then

$$
f_{F_{1}, \ldots, F_{k}}\left(u^{\prime}\right)=\left|\operatorname{det}\left(e_{1}, \ldots, e_{n-1}, u^{\prime}\right)\right|=\left|\left\langle y_{F_{1}, \ldots, F_{k}}, u^{\prime}\right\rangle\right|
$$

with $y_{F_{1}, \ldots, F_{k}}:=e_{1} \times \cdots \times e_{n-1}$ (vector product). This vector is orthogonal to $N$. The scalar product $\left\langle y_{F_{1}, \ldots, F_{k}}, u^{\prime}\right\rangle$ does not vanish in int $Z_{j}$, hence we may assume that $f_{F_{1}, \ldots, F_{k}}\left(u^{\prime}\right)=\left\langle y_{F_{1}, \ldots, F_{k}}, u^{\prime}\right\rangle$ for $u^{\prime} \in \operatorname{int} Z_{j}$. Now it is clear that

$$
v_{j}=v_{m_{1}, \ldots, m_{k}}\left(P_{1}, \ldots, P_{k}, w\right)+V_{m_{1}}\left(F_{1}\right) \cdots V_{m_{k}}\left(F_{k}\right) y_{F_{1}, \ldots, F_{k}} \neq v_{i},
$$

that $N$ is the normal cone of the edge of $T_{m_{1}, \ldots, m_{k}}\left(P_{1}, \ldots, P_{k}\right)$ connecting $v_{i}$ and $v_{j}$, and that this edge has length

$$
V_{m_{1}}\left(F_{1}\right) \cdots V_{m_{k}}\left(F_{k}\right)\left\|y_{F_{1}, \ldots, F_{k}}\right\|=\left[F_{1}, \ldots, F_{k}\right] V_{m_{1}}\left(F_{1}\right) \cdots V_{m_{k}}\left(F_{k}\right) .
$$

We collect this information on the edges of $T_{m_{1}, \ldots, m_{k}}\left(P_{1}, \ldots, P_{k}\right)$, which for $k=2$ was essentially first obtained in [3].

Theorem 5.3. Let $m_{1}, \ldots, m_{k} \in\{1, \ldots, n\}$ and $m_{1}+\cdots+m_{k}=(k-1) n+1$. Let $P_{1}, \ldots, P_{k} \subset \mathbb{R}^{n}$ be polytopes satisfying Assumption 5.2. Then to each $k$-tuple $F_{1} \in \mathcal{F}_{m_{1}}\left(P_{1}\right), \ldots, F_{k} \in \mathcal{F}_{m_{k}}\left(P_{k}\right)$ of faces there corresponds a set $\left\{E_{1}, \ldots, E_{m}\right\}$, $m \geq 1$, of parallel edges of $T_{m_{1}, \ldots, m_{k}}\left(P_{1}, \ldots, P_{k}\right)$ of length

$$
\left[F_{1}, \ldots, F_{k}\right] V_{m_{1}}\left(F_{1}\right) \cdots V_{m_{k}}\left(F_{k}\right)
$$

such that

$$
N\left(P_{1}, F_{1}\right)+\cdots+N\left(P_{k}, F_{k}\right)=\bigcup_{r=1}^{m} N\left(T_{m_{1}, \ldots, m_{k}}\left(P_{1}, \ldots, P_{k}\right), E_{r}\right),
$$

and these are all the edges of $T_{m_{1}, \ldots, m_{k}}\left(P_{1}, \ldots, P_{k}\right)$. 


\section{An Extremal Property of Simplices}

As carried out in [16] and [3] for $k=2$, the expansion (8) extends to general convex bodies, by approximation and continuity. Hence, for convex bodies $K_{1}, \ldots, K_{k}$ in $\mathbb{R}^{n}$, there are convex bodies $T\left(K_{1}, \ldots, K_{k}\right)$ and $T_{m_{1}, \ldots, m_{k}}\left(K_{1}, \ldots, K_{k}\right)$ such that

$$
\begin{gathered}
\int_{\mathbb{R}^{n}} \cdots \int_{\mathbb{R}^{n}} h^{*}\left(\left(K_{1}+x_{1}\right) \cap \cdots \cap\left(K_{k-1}+x_{k-1}\right) \cap K_{k}, \cdot\right) d x_{1} \cdots d x_{k-1} \\
=h\left(T\left(K_{1}, \ldots, K_{k}\right), \cdot\right)
\end{gathered}
$$

and

$$
T\left(\lambda_{1} K_{1}, \ldots, \lambda_{k} K_{k}\right)=\sum_{\substack{m_{1}, \ldots, m_{k}=1 \\ m_{1}+\cdots+m_{k}=(k-1) n+1}}^{n} \lambda_{1}^{m_{1}} \cdots \lambda_{k}^{m_{k}} T_{m_{1}, \ldots, m_{k}}\left(K_{1}, \ldots, K_{k}\right)
$$

for $\lambda_{1}, \ldots, \lambda_{k} \geq 0$.

The translation mixture $T(K, K)$ of a convex body $K$ with itself can be compared with $K$ : there is a vector $t$ such that the inclusion

$$
T(K, K) \supseteq \frac{1}{n+1} V_{n}(\mathrm{D} K) K+t
$$

holds; here $\mathrm{D} K=K-K$ is the difference body of $K$. Equality in (22) holds if and only if $K$ is a simplex. The inclusion (22) is a special case of the following more general result. For convex bodies $K_{1}, \ldots, K_{k} \subset \mathbb{R}^{n}(k \geq 2)$, let

$$
\begin{aligned}
\mathrm{D}\left(K_{1}, \ldots, K_{k}\right) & \\
& :=\left\{\left(x_{1}, \ldots, x_{k-1}\right) \in\left(\mathbb{R}^{n}\right)^{k-1}:\left(K_{1}+x_{1}\right) \cap \cdots \cap\left(K_{k-1}+x_{k-1}\right) \cap K_{k} \neq \emptyset\right\}
\end{aligned}
$$

(so that $\mathrm{D}(K, K)=\mathrm{D} K)$.

Theorem 6.1. For convex bodies $K_{1}, \ldots, K_{k} \subset \mathbb{R}^{n}(k \geq 2)$, the inclusion

$$
T\left(K_{1}, \ldots, K_{k}\right) \supseteq \frac{1}{(k-1) n+1} V_{(k-1) n}\left(\mathrm{D}\left(K_{1}, \ldots, K_{k}\right)\right)\left(K_{1} \cap \cdots \cap K_{k}\right)+t
$$

holds with a suitable translation vector $t$. Equality holds if and only if $K_{1}, \ldots, K_{k}$ are identical simplices.

The proof will be obtained by a modification of the proof of the difference body inequality, as reproduced in Section 7.3 of [8], and extended in [7]. Let convex bodies $K_{1}, \ldots, K_{k}$ be given. We abbreviate $\mathrm{D}\left(K_{1}, \ldots, K_{k}\right)$ by $D$ and define

$$
K(X):=\left(K_{1}+x_{1}\right) \cap \cdots \cap\left(K_{k-1}+x_{k-1}\right) \cap K_{k}
$$

for $X=\left(x_{1}, \ldots, x_{k-1}\right) \in\left(\mathbb{R}^{n}\right)^{k-1}$. Then $D=\left\{X \in\left(\mathbb{R}^{n}\right)^{k-1}: K(X) \neq \emptyset\right\}$. As on p. 269 of [7], one shows that

$$
(1-\lambda) K\left(X_{1}\right)+\lambda K\left(X_{2}\right) \subseteq K\left((1-\lambda) X_{1}+\lambda X_{2}\right)
$$


for $X_{1}, X_{2} \in D$ and $0 \leq \lambda \leq 1$. The set $D$ is convex and compact. Without loss of generality we assume that $K_{1} \cap \cdots \cap K_{k} \neq \emptyset$, then $O \in D$, where $O$ denotes the zero vector of $\left(\mathbb{R}^{n}\right)^{k-1}$. Now let $X=\left(x_{1}, \ldots, x_{k-1}\right) \in D \backslash\{O\}$. The ray in $\left(\mathbb{R}^{n}\right)^{k-1}$ through $X$ with endpoint $O$ intersects $D$ in a segment $\left[O, Y_{X}\right]$, and we can write $X=\lambda_{X} Y_{X}$ with $0<\lambda_{X} \leq 1$. By (24),

$$
\left(1-\lambda_{X}\right) K(O)+\lambda_{X} K\left(Y_{X}\right) \subseteq K\left(\lambda_{X} Y_{X}\right)=K(X) .
$$

Since $Y_{X} \in D$, the intersection $K\left(Y_{X}\right)$ is a nonempty convex body (with empty interior, since $Y_{X} \in \operatorname{bd} D$ ); let $s(X)$ be its Steiner point. Let $u \in \mathbb{R}^{n}$ be a given vector.Then (25) gives

$$
\begin{aligned}
h\left(\left(K_{1}+x_{1}\right)\right. & \left.\cap \cdots \cap\left(K_{k-1}+x_{k-1}\right) \cap K_{k}, u\right) \\
\geq & \left(1-\lambda_{X}\right) h\left(K_{1} \cap \cdots \cap K_{k}, u\right)+\lambda_{X}\langle s(X), u\rangle
\end{aligned}
$$

and thus

$$
\begin{aligned}
h\left(\tilde{T}\left(K_{1}, \ldots, K_{k}\right), u\right) & \\
& :=\int_{D} \cdots \int_{D} h\left(\left(K_{1}+x_{1}\right) \cap \cdots \cap\left(K_{k-1}+x_{k-1}\right) \cap K_{k}, u\right) d x_{1} \cdots d x_{k-1} \\
& \geq h\left(K_{1} \cap \cdots \cap K_{k}, u\right) \int_{D}\left(1-\lambda_{X}\right) d X+\int_{D} \lambda_{X}\langle s(X), u\rangle d X
\end{aligned}
$$

where $d X$ indicates integration with respect to the $((k-1) n)$-dimensional Lebesgue measure in $\left(\mathbb{R}^{n}\right)^{k-1}$. The last integral is of the form $\langle z, u\rangle$ with a vector $z \in \mathbb{R}^{n}$ independent of $u$. Since $u \in \mathbb{R}^{n}$ was arbitrary and $T\left(K_{1}, \ldots, K_{k}\right)$ is a translate of $\tilde{T}\left(K_{1}, \ldots, K_{k}\right)$, we deduce that there is a vector $t$ so that

$$
T\left(K_{1}, \ldots, K_{k}\right) \supseteq c\left(K_{1} \cap \cdots \cap K_{k}\right)+t
$$

with

$$
c=\int_{D}\left(1-\lambda_{X}\right) d X
$$

Introducing polar coordinates in $\mathbb{R}^{(k-1) n}$, one finds that

$$
c=\frac{1}{(k-1) n+1} V_{(k-1) n}(D) \text {. }
$$

This proves the inequality (23) (see the proof of Lemma 7.3.2 in [8]).

Suppose that (23) holds with equality. Then equality holds in (26) for all $u \in \mathbb{R}^{n} \backslash\{0\}$ and all $X \in D$. This implies that equality holds in (25), for all $X \in D$, and that the set $K\left(Y_{X}\right)$ is one-pointed for all $X$. Hence, $K(X)$ is homothetic to $K(O)$ whenever $\operatorname{dim} K(X)=n$, and the homothety factor is less than 1 if $X \neq O$. In particular, let $M:=K(O), K:=K_{1}, L:=K_{2} \cap \cdots \cap K_{k}$. The choice $X=\left(x_{1}, 0, \ldots, 0\right)$ shows that $\left(K+x_{1}\right) \cap L$, if $n$-dimensional, is homothetic to $M$. Now it follows from a result of Soltan [11] that $K, L, M$ are homothetic simplices. In a similar way, one obtains that 
$K_{1}, \ldots, K_{k}$ are all homothetic to the simplex $K(O)$. Suppose that one of the homothetic simplices $K_{1}, \ldots, K_{k}$, say $K_{1}$, is not identical to their intersection $K(O)$. Then $X=$ $\left(x_{1}, 0, \ldots, 0\right) \neq O$ can be chosen so that $K(X) \supseteq K(O)$, contradicting the fact that $K(X)$ must be homothetic to $K(O)$ with homothety factor less than 1 . Hence, $K_{1}, \ldots, K_{k}$ all coincide with the same simplex.

\section{References}

1. L.J. Billera and B. Sturmfels, Fiber polytopes, Ann. Math. 135 (1992), 527-549.

2. P. Goodey and W. Weil, Translative integral formulae for convex bodies, Aequationes Math. 34 (1987), 64-77.

3. P. Goodey and W. Weil, Translative and kinematic formulae for support functions II, Preprint No. 01/19, Universität Karlsruhe, 2001, to appear in Geom. Dedicata.

4. P. McMullen, Valuations and dissections, in Handbook of Convex Geometry, vol. B (P.M. Gruber and J.M. Wills, eds.), pp. 933-988, Elsevier, Amsterdam, 1993.

5. J. Rataj, Translative and kinematic formulae for curvature measures of flat sections, Math. Nachr. 197 (1999), 89-101.

6. J. Rataj and M. Zähle, Mixed curvature measures for sets of positive reach and a translative integral formula, Geom. Dedicata 57 (1995), 259-283.

7. R. Schneider, Eine Verallgemeinerung des Differenzenkörpers, Monatsh. Math. 74 (1970), 258-272.

8. R. Schneider, Convex Bodies - the Brunn-Minkowski Theory, Cambridge University Press, Cambridge, 1993.

9. R. Schneider, Mixed functionals of convex bodies, Discrete Comput. Geom. 24 (2000), 527-538.

10. R. Schneider and W. Weil, Translative and kinematic integral formulae for curvature measures, Math. Nachr. 129 (1986), 67-80.

11. V. Soltan, A characterization of homothetic simplices. Discrete Comput. Geom. 22 (1999), 193-200.

12. W. Weil, Translative integral geometry, in Geobild' 89 (A. Hübler et al., eds.), pp. 75-86, Akademie-Verlag, Berlin, 1989.

13. W. Weil, Iterations of translative integral formulae and non-isotropic Poisson processes of particles, Math. Z. 205 (1990), 531-549.

14. W. Weil, Lectures on translative integral geometry and stochastic geometry of anisotropic random geometric structures, in Atti Primo Convegno Ital. Geom. Integrale, Rend. Sem. Mat. Messina (2) 13 (1990), 79-97.

15. W. Weil, Support functions on the convex ring in the plane and support densities for random sets and point processes, Rend. Circ. Mat. Palermo (2) Suppl. 35 (1994), 323-344.

16. W. Weil, Translative and kinematic integral formulae for support functions, Geom. Dedicata 57 (1995), 91-103.

17. W. Weil, Mixed measures and functionals of translative integral geometry, Math. Nachr. 223 (2001), 161-184.

Received May 31, 2002, and in revised form September 18, 2002, and September 26, 2002.

Online publication January 30, 2003. 